\title{
Communicating the intention of an automated vehicle to pedestrians: The contributions of eHMI and vehicle behavior
}

https://doi.org/10.1515/itit-2020-0025

Received July 14, 2020; revised October 9, 2020; accepted October 14, 2020

Abstract: External Human-Machine Interfaces (eHMIs) are expected to bridge the communication gap between an automated vehicle (AV) and pedestrians to replace the missing driver-pedestrian interaction. However, the relative impact of movement-based implicit communication and explicit communication with the aid of eHMIs on pedestrians has not been studied and empirically evaluated. In this study, we pit messages from an eHMI against different driving behaviors of an AV that yields to a pedestrian to understand whether pedestrians tend to pay more attention to the motion dynamics of the car or the eHMI in making road-crossing decisions. Our contributions are twofold: we investigate (1) whether the presence of eHMIs has any objective effect on pedestrians' understanding of the vehicle's intent, and (2) how the movement dynamics of the vehicle affect the perception of the vehicle intent and interact with the impact of an eHMI. Results show that (1) eHMIs help in convincing pedestrians of the vehicle's yielding intention, particularly when the speed of the vehicle is slow enough to not be an obvious threat, but still fast enough to raise a doubt about a vehicle's stopping intention, and (2) pedestrians do not blindly trust the eHMI: when the eHMI message and the vehicle's movement pattern contradict, pedestrians fall back to movement-based cues. Our results imply that when explicit communication (eHMI) and implicit communication (motion-dynamics and kinematics) are in alignment and work in tandem, communication of the AV's yielding intention can be facilitated most

\footnotetext{
*Corresponding author: Debargha Dey, Eindhoven University of Technology, Groene Loper 3, 5612 AE Eindhoven, The Netherlands, e-mail: d.dey@tue.nl, ORCID:

https://orcid.org/0000-0001-9266-0126

Andrii Matviienko, Technical University of Darmstadt, Hochschulstraße 10, 64289 Darmstadt, Germany, e-mail: matviienko@tk.tu-darmstadt.de

Melanie Berger, Bastian Pfleging, Marieke Martens, Jacques Terken, Eindhoven University of Technology, Groene Loper 3, 5612 AE Eindhoven, The Netherlands, e-mails: m.berger@tue.nl, b.pfleging@tue.nl,m.h.martens@tue.nl, j.m.b.terken@tue.nl
}

effectively. This insight can be useful in designing the optimal interaction between AVs and pedestrians from a usercentered design perspective when driver-centric communication is not available.

Keywords: Automated vehicles, eHMI, Pedestrian, Vulnerable road user, VRU, Explicit communication, Implicit communication

ACM CCS: Human-centered computing $\rightarrow$ Interaction techniques Human-centered computing $\rightarrow$ Ubiquitous and mobile computing systems and tools

\section{Introduction}

There are different schools of thought when it comes to effectively facilitating the interactions between an automated vehicle (AV) and Vulnerable Road Users (VRU) such as pedestrians. One recommends the use of eHMIs as the solution to mitigate the uncertainty that arises from a pedestrians' inability to communicate with a 'driver' inside: In negotiation situations when the paths of AVs and pedestrians intersect, an eHMI shows promise in resolving ambiguity and increasing pedestrians' feelings of trust and safety in AVs [4, 14, 16, 17, 18]. However, there exist other research that found no significant effect of an eHMI on pedestrians' road crossing decisions [3]. The other school of thought posits that the motion dynamics of the vehicle can act as a form of 'implicit communication' through movement patterns, and are enough to convey the intentions of an AV in traffic: Some works suggest that such forms of implicit communication is enough and further augmentation with eHMI is discouraged due to potential 'griefing behavior' (mild form of bullying) from bystanders [19], especially in the early stages of integration of automated driving technology in traffic. Given the empirical evidence supporting arguments both for eHMIs, and against eHMIs in favor of vehicle behavior-based communication, it is important to understand the relative impact of both in order to design an effective and usable interaction experience for AV-pedestrian interaction. 
There are contradicting reports regarding pedestrians' perception of automated vehicles. Prior research suggests that in general there is a hesitation and mistrust towards automated driving technology [2, 21]. However, other works suggest that an 'innocent until proven guilty' exists with pedestrians with regards to automated vehicles and they are not hesitant to trust automated vehicles in general [22] and eHMI systems in particular [15]. Although past research related to the efficacy of eHMI shows mostly positive results, the role of eHMIs in facilitating AVpedestrian interactions is not yet well-understood. Interaction between AVs and pedestrians in traffic is a complex problem that depends on many factors. Prior works have studied the efficacy of eHMIs in controlled and specific behavior conditions of the vehicle. However, a vehicle can yield while braking in different ways, which translate to different perceived movement patterns. To design an effective user experience, it is critical to know whether the efficacy of an eHMI unilaterally holds over different yielding behaviors.

Additionally, previous research with manually operated vehicles showed that pedestrians exhibit a specific gaze pattern when observing oncoming vehicles [11]. The study revealed that pedestrians' gaze tends to shift from the environment and the road surface in front of the vehicle when it is far away, towards the bumper, grill, hood, and windshield of the vehicle as it approaches closer. Pedestrians particularly fixated on the windshield, which was seen as an indication that they sought confirmatory information about the driver's intention. It is interesting to see if the presence of an eHMI is able to resolve this ambiguity or whether pedestrians still seek additional information. Besides, given that most eHMI design concepts are light-based, emissive displays [6], it is also important to understand whether the presence of an eHMI causes a distractingly high attention demand in pedestrians relative to when there is no eHMI present.

To this end, we used a light-bar eHMI concept and evaluated the interaction behavior of pedestrians to a simulated AV that exhibited three different yielding behaviors: (1) Gentle braking, (2) Early braking, (3) Aggressive braking, and one non-yielding behavior: (4) Constant speed. In a controlled real-world study $(N=24)$, we compared the pedestrians' gaze patterns and willingness to cross in response to these behaviors, with and without the eHMI.

Our results show that an eHMI is able to resolve pedestrians' ambiguity and clarify a yielding AV's intention, particularly in low-speed conditions. The effect of eHMI is not pronounced if the speed of the vehicle is high. Additionally, if an eHMI communicates a yielding message which is not in concurrence with the vehicle's behavior, pedestrians do not blindly trust the eHMI, but instead fall back on vehicle behavior-related cues. Besides, there is no evidence that a light bar eHMI attracts excessive attention or causes distraction in pedestrians.

We posit that the effects of eHMI and vehicle behavior are not straightforward and are interdependent. For an optimal interaction, the explicit communication from an eHMI must align with the implicit communication with the vehicle's behavior.

\section{Contribution statement}

The findings from our real-world study contribute to the body of knowledge by showing that the effectiveness of an eHMI is not absolute, and depends on the speed and behavior of the AV. However, if used correctly in conjunction with the right speed and behavior, it shows promise of improving the user experience of AV-pedestrian interaction. This insight provides a foundation for future work in determining the optimal interaction paradigm.

\section{2 eHMI concept}

The eHMI concept used in our study is an adaptation of prior 'light band' concepts [9, 13, 24]. The eHMI is a one-dimensional light bar fully integrated into the car body across the headlights and the grill as shown in Figure 1. When the AV drives (cruises) in automated mode, it glows in a solid, turquoise color. When intending to yield, the eHMI communicates this through two small light segments that originate from the edges and animate sideways towards the middle until they disappear. This animation repeats as the AV continues to yield. The animation pattern was inspired by Dey et al. [9] as it was thought to be an abstract way of mapping to a gesture of a driver's hand motion asking a pedestrian to cross in front of the car. When the car intends to start driving again, the light bar returns to a steady glowing state.

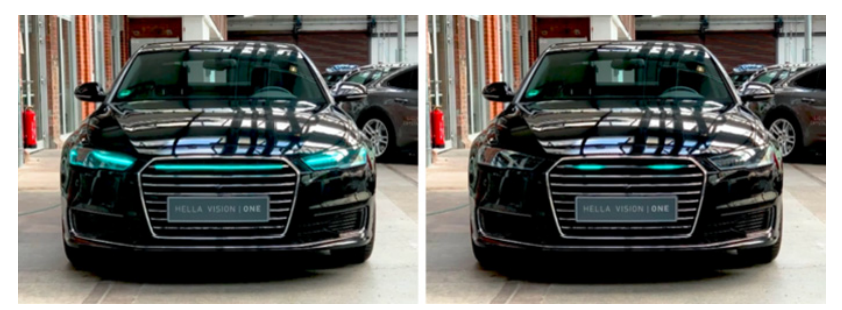

Figure 1: The eHMI concept. Left: Cruising, Right: Yielding. 
Prior work by Dey et al. [6] suggests that eHMI concepts ought to be categorized based on their properties and attributes across 18 dimensions to aid a unified approach towards eHMI concept proposal and evaluation. Consequently, the concept used in our study is coded in Appendix B according to the classification taxonomy.

\section{Research question \& hypotheses}

We used the eHMI concept to evaluate the following research questions:

RQ1: For the same driving behavior, does the presence of an eHMI on a yielding AV increase the pedestrians' willingness to cross?

Although there is currently no consensus on the kind of eHMI that is ideally suited for AV-pedestrian communication, prior research indicate that eHMIs can be effective in clarifying the intention of an $\operatorname{AV}[1,4,13,14,16]$. Therefore, we hypothesize that an eHMI will elucidate the vehicle's intention and for a yielding vehicle, pedestrians will show a willingness to cross more quickly than without an eHMI (H1).

RQ2: When the driving behavior of the AV appears to contradict a yielding message from the eHMI, which source of information do pedestrians rely on?

Prior work found arguments in both directions. Some showed that pedestrians tend to start off with a high degree of trust towards eHMIs, and that even if the trust is broken, they are quick to regain it [15]. However, others found that the vehicle behavior plays a primary role in modulating pedestrians' behavior in interaction situations with vehicles $[10,8,19,20]$. However, we believe that when the message from an eHMI does not correspond with a vehicle's behavior, 'what the AV is doing' is a more important factor in determining safety than 'what the eHMI is saying'. Therefore, we expect that if an eHMI message contradicts the behavior of the AV, pedestrians will fall back on the vehicle behavior based cues (H2).

RQ3: When a light-based eHMI is active on an AV, does it draw more attention to the corresponding area of the vehicle compared to when the eHMI is inactive?

Adding any external stimulus to an object has the potential of attracting attention due to increased salience. When a light-based eHMI is on, it is possible that pedestrians will be drawn to look more at the headlights-and-grill area where the eHMI is located compared to when the eHMI is off, either due to its novelty or its salience. As a result, we hypothesize that when an eHMI is on, pedestrians will fixate on it longer than the corresponding location of the $\mathrm{AV}$ when the eHMI is off (H3).

\section{Method}

We evaluated the efficacy of the eHMI and the effects of different braking behaviors on pedestrians' road-crossing willingness in a real-world outdoor controlled experiment. The experiment was reviewed and approved by the ethical review board of the researchers' institution.

\subsection{Task}

In this controlled outdoor experiment, the participants (who assume the role of a pedestrian who wants to cross a road) had to stand on the edge of a pavement next to a road and watch an $\mathrm{AV}$ approach them while exhibiting different driving and communication behaviors. While observing the approaching $\mathrm{AV}$, the participant indicated their willingness to cross the road in real time. The participants were not asked to actually cross the road, but rather indicate their willingness to cross the road using a slider input device.

\subsection{Apparatus and study setup}

This experiment was conducted in collaboration with HELLA. ${ }^{1}$ The platform used for this experiment was the Hella Vision One concept vehicle, which was an Audi A6 C7 with the headlights and the grills modified to reflect a "light bar" eHMI (see Figure 2) that glowed with a turquoise (bluish-green) color as explained in Section 2. The location of the pedestrian was at the curbside of a straight road that was free from any other traffic or road users. The interaction took place at a location where there was no intersection or pedestrian crossing. This was done to ensure that the decision whether or not to cross the road is a direct result of the consideration of the car's behavior and/ or communication, and not of an expectation of right of way. The setup of the experiment is shown in Figure 2.

We used a Ghost Driver Wizard-of-Oz setup to hide the driver under a 'seat suit' and to create an illusion of an automated vehicle [23]. The high-fidelity nature of the eHMI

1 https://www.hella.com/, last access 2020-07-09. 


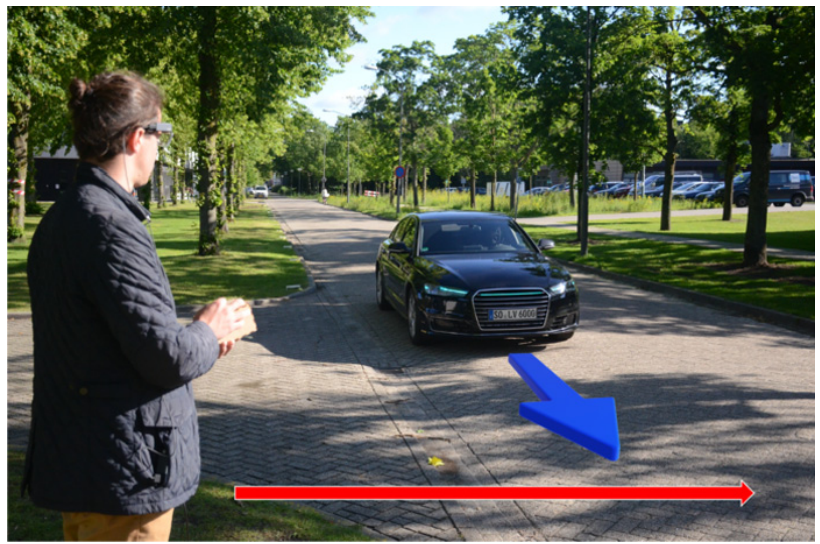

Figure 2: Experiment setup: The pedestrian stands on the edge of the road on the pavement wearing a mobile eye tracker and holding the slider input device and indicates their willingness to cross in real time as the $A V$ approaches them. The blue and red arrows represent the path of the $A V$ and the pedestrian respectively.

prototype in the Hella Concept One vehicle, as well as the Wizard-of-Oz setup allowed us to create an experience of an automated vehicle for the participants with a high level of immersion. The experiment was carried out during daytime and on a clean road that was devoid of debris, pooled water, etc. (to avoid any confounding element that the car might be slowing down for any anomaly on the road).

To determine the participants' gaze patterns during the experiment, we used a mobile eye tracker (Tobii ${ }^{2}$ Pro Glasses 2) which was calibrated with each participant at the beginning of the experiment. To ensure a successful calibration, the experimenter asked the participant to look at 3 different objects in the environment at pre-determined distances $(10 \mathrm{~m}, 30 \mathrm{~m}$, and $50 \mathrm{~m})$, and validated whether the fixation was recorded correctly.

The focus of this study was to investigate how pedestrians interact with automated vehicles that exhibit different braking behaviors while yielding, and the effect of explicit communication through an eHMI. One of the goals of this study was to investigate how eHMI and vehicle behavior interact with each other, particularly if they present apparently contradicting messages. In this context, the experiment included two eHMI conditions: presence or absence of the eHMI (eHMI/ no eHMI), and four behavior conditions (Gentle braking, Early braking, Aggressive braking, and Constant speed/No braking). While a car can yield to a pedestrian in different ways by employing different braking patterns and speed profiles, we investigated three

2 https://www.tobiipro.com/product-listing/tobii-pro-glasses-2, last access 2020-07-09.
Table 1: Study design - The AV exhibited 4 driving behaviors, each of them with or without activating the eHMI. In a within-subjects design, the participants experienced these 8 conditions in a counterbalanced order.

\begin{tabular}{cll}
\hline Trial \# & $\begin{array}{l}\text { eHMI } \\
\text { Condition }\end{array}$ & Behavior \\
\hline 1 & & Gentle braking $(50 \mathrm{~km} / \mathrm{h} \searrow 0 \mathrm{~km} / \mathrm{h})$ \\
2 & No & Early braking $(50 \mathrm{~km} / \mathrm{h} \searrow 20 \mathrm{~km} / \mathrm{h} \searrow 0 \mathrm{~km} / \mathrm{h})$ \\
3 & eHMI & Aggressive braking $(50 \mathrm{~km} / \mathrm{h} \searrow 0 \mathrm{~km} / \mathrm{h})$ \\
4 & & Constant speed $(50 \mathrm{~km} / \mathrm{h} \mathrm{constant)}$ \\
\hline 5 & & Gentle braking $(50 \mathrm{~km} / \mathrm{h} \searrow 0 \mathrm{~km} / \mathrm{h})$ \\
6 & eHMI & Early braking $(50 \mathrm{~km} / \mathrm{h} \searrow 20 \mathrm{~km} / \mathrm{h} \searrow 0 \mathrm{~km} / \mathrm{h})$ \\
7 & & Aggressive braking $(50 \mathrm{~km} / \mathrm{h} \searrow 0 \mathrm{~km} / \mathrm{h})$ \\
8 & & Constant speed $(50 \mathrm{~km} / \mathrm{h}$ constant $)$ \\
\hline
\end{tabular}

distinctly different yielding behaviors to simplify the scope of this study. The yielding behaviors were also selected to specifically conform to or contrast the eHMI message. To avoid learning effects (that the car yielded every time), we added a non-yielding behavior in the study where the car drove by at constant speed and did not stop. The two eHMI conditions and the four behavior conditions led to a total number of $2 \times 4=8$ trials. These 8 trials were counterbalanced to avoid any learning effects. The study design is shown in table 1.

In each condition, the car approached from a distance of $200 \mathrm{~m}$ at $50 \mathrm{~km} / \mathrm{h}$ (standard city driving speed in Europe). In the yielding conditions, it came to a full stop at $5 \mathrm{~m}$ before the pedestrian. The three different yielding behaviors exhibited different braking characteristics:

Gentle braking: At a distance of $45 \mathrm{~m}$ away from the pedestrian, the car started to brake steadily, to indicate a deliberate but smooth yielding behavior, resulting in a total braking distance of $40 \mathrm{~m}$. This was done to emulate a deceleration rate of $2.4 \mathrm{~m} / \mathrm{s}^{2}$ which was found as the deceleration rate for average and common braking by previous research [5]. This condition was therefore treated as a representative of a 'normal' braking pattern which would ideally correspond with a 'yielding' message from an eHMI.

Early braking: At a distance of $45 \mathrm{~m}$ away from the pedestrian, the car braked hard and slowed down quickly to a speed of $20 \mathrm{~km} / \mathrm{h}$ within a distance of $\sim 16 \mathrm{~m}$ (deceleration of $5.17 \mathrm{~m} / \mathrm{s}^{2}$ ). The vehicle covered the rest of the distance slowly until coming to a complete stop. This was done to show a more 'deliberate' yielding behavior where the vehicle showed with its movement patterns and 'body language' in advance that it was slowing down. This condition was chosen as a candidate because it was shown in previous research to 
have a positive effect in conveying to pedestrians the yielding intention of the vehicle and aiding pedestrians' decision-making process [12]. We wanted to test the effectiveness of this braking behavior to yield, particularly when combined with an eHMI.

Aggressive braking: At a distance of $24 \mathrm{~m}$ away from the pedestrian, the car braked hard. In this aggressive yielding behavior, the total braking distance was $19 \mathrm{~m}$. This was done to emulate a deceleration rate of $5.17 \mathrm{~m} / \mathrm{s}^{2}$ which was found as the maximum deceleration rate for hard braking by previous research [5]. This condition was therefore treated as a representative of behavior that would initially seem to contradict a 'yielding' message from an eHMI.

The participants experienced each behavior with and without the eHMI active. When the eHMI was not active, the daytime running lights of the vehicle were switched off so as not to cause any distraction. When the eHMI was active, the eHMI showed the 'cruising' pattern at the beginning of each trial, and triggered the 'yielding' pattern at a distance of $45 \mathrm{~m}$ for each of the three yielding behaviors. This means that for the Gentle braking and Early braking conditions, the eHMI showed the yielding message the moment the vehicle started to brake, and for the Aggressive braking condition, the eHMI showed that the vehicle intended to yield before the vehicle had actually started to brake. This was done to ensure that participants had enough time to consider the eHMI message and not just be influenced by the vehicle behavior. However, the study was designed in a way that the eHMI and the vehicle behavior - even if they apparently contrasted each other (particularly in the Aggressive braking condition) - never fully contradicted, and the eHMI never communicated that the vehicle was going to yield when it did not. This was done to ensure that the pedestrians would not lose trust in the eHMI and that their responses would stem from the eHMI and the vehicle behavior organically, instead of being confounded by an element of broken trust. When the car came to a complete stop, the eHMI switched back to the 'cruising' pattern after a wait of 3 seconds, and started driving away for the subsequent trial.

Each stimulus consisted of an approach of the car from when it was approximately $200 \mathrm{~m}$ away until either 3 seconds after having stopped for the pedestrian, or until having passed the pedestrian without stopping. We recorded the pedestrians' willingness-to-cross to the yielding car from when the car was 12 seconds away from the pedestrian. For a yielding vehicle, we measured the pedestrians' willingness-to-cross relative to the 'Time-to-stop' of the vehicle, which we defined as the moment when the vehicle comes to a complete stop in front of the pedestrian. For a non-yielding vehicle, we measured relative to the 'Timeto-arrival' of the vehicle, which we defined as the moment when the front bumper of the vehicle reached the pedestrian's location.

To record the pedestrians' willingness to cross as a function of time in terms of the vehicle's time-to-stop or time-to-arrival, we used a slider device as input device as proposed by Walker et al. [25]. The participant could move the slider to indicate their willingness to cross the road. The two ends of the slider were mapped to 0 and 100 (corresponding to no willingness to cross, and total willingness to cross), and the device recorded inputs at a rate of $10 \mathrm{~Hz}$. We also instructed the participants that the continuum of the slider in between the ends can be used to express ambiguity regarding their decision.

\subsection{Procedure}

Three days before the experiment was scheduled for each participant, we sent them an online tutorial to acquaint them with the functioning of the eHMI, and what a particular signal by the eHMI meant regarding the intention of the car. This online tutorial consisted of a number of videos (with a total duration of approximately 1:05 min) that demonstrated and explained the behavior of the eHMI within the context of the operating cycle of the car (cruising, yielding, beginning to drive). This step was used as a point of communicating the functionality of the eHMI to the participant and to exclude the (potential lack of) intuitiveness of the eHMI as a possible confounding factor. Each participant was asked to follow the online tutorial to familiarize themselves with the message of the eHMI before participating in the experiment.

On the day of the experiment, after each participant gave their informed consent, the experimenters verified that the participant had gone through the online tutorial. Subsequently, they were requested to answer several questions regarding their comprehension of the functionality of the eHMI using a questionnaire before the start of the experiment process. This was done to ascertain that they had understood and learned the functionality of the eHMI. Each participant answered each question correctly at the first attempt, indicating an understanding of the concept of the eHMI. This ensured that the results of their responses were an accurate measure of the efficacy of the eHMI and not of its intuitiveness.

Subsequently, we guided the participant to the predefined outdoor location of the experiment where the AVpedestrian interaction would take place. The participant 
stood approximately $1 \mathrm{~m}$ from the edge of the pavement. We asked them to imagine that they would cross to the other side of the road, while they observed the AV which approached them from their left (see Figure 2). Once the participant took their position, the experimenter(s) helped them to put on the mobile eye tracker and calibrated it to ensure accuracy of fixation data. Subsequently, the experimenter(s) handed them the slider input device and asked them to hold it comfortably in their hand, consider the approaching $\mathrm{AV}$, and indicate their willingness to cross in real time.

Before the measured trials began, the participant had the opportunity to experience two practice trials to familiarize themselves with the setup and the slider input device. The two stimuli used for the practice trial were the Constant speed and Gentle braking behaviors with 'NoeHMI' condition, and the participants experienced each behavior once in a randomized order. After the practice trials, the participants were asked if they understood the task and were comfortable with continuing with the study. Each participant gave a positive response, and was allowed to continue with the measured trials.

The experiment concluded with a short semistructured interview/discussion with the participant regarding how they perceived the crossing scenarios. The entire experiment took approximately 30 minutes, and each participant was compensated for their time with $€ 10$.

\subsection{Measures}

This study incorporated three independent variables: the vehicle behavior and the eHMI condition as explained in section 4.2, and time until the vehicle stopped or arrived at the pedestrian's location (Time to Arrival - TTA; Time to Stop - TTS). Two different dependent variables were used as measures to evaluate the effect of eHMIs on pedestrians' road crossing behaviors. Firstly, we used the Willingness to Cross data from the slider input device as an objective surrogate measure for the pedestrians' feeling of safety around the automated vehicle [25]. Secondly, we used the Gaze Behavior data acquired from the mobile eye tracker to determine how the fixation pattern of the pedestrians varied as the $\mathrm{AV}$ approached, and how it differed between the eHMI being triggered and the eHMI being switched off.

\subsection{Participants}

We conducted the study with university students and staff whom we recruited via different channels including the university participation database, social media, and word of mouth $(N=26,16$ male, 10 female; mean age = 26.35 years; $S D=4.13$ years). We only recruited users who had no color-blindness, no mobility issues, and had normal or corrected-to-normal vision. We implemented a within-subjects setup across the 8 conditions as shown in Table 1. We lost the willingness to cross data from two participants due to technical issues. Thus, we used a sample of $N=24$ (15 male, 9 female; mean age $=26.21$ years; $\mathrm{SD}=3.74$ years) for the analysis.

Results of pedestrians' interactions with an eHMI cannot be generalized across all conditions unless the evaluations of the performance of the eHMI is also conducted in all conditions. However, as a first step, we evaluated the effects of eHMI in a specific set of conditions and put forward the findings. We highlight the parameters of our evaluation in congruence with the taxonomy introduced in [6] in Table 5.

\section{Results}

We analyzed each measure (willingness to cross, and gaze behavior) separately, and we report the results in the following sections.

\subsection{Willingness to cross}

For each behavior condition with and without eHMI, we extracted the willingness-to-cross values in $0.5 \mathrm{~s}$ intervals. The pedestrians' willingness to cross with and without the eHMI as a function of time (Time to stop: until the car comes to a complete stop, or Time to arrival: until the front bumper reaches the position of the pedestrian) for each of the 4 behaviors are shown in Figures 3 to 6 .

Subsequently, for each behavior, we conducted a repeated-measures ANOVA across the time (Time to Arrival - TTA, or Time to Stop - TTS) from $11 \mathrm{~s}$ until $+2 \mathrm{~s}$, and the eHMI (see Table 2).

Results show that as expected, Time had a highly significant effect on pedestrians' willingness to cross in all behaviors - it varied as the vehicle came closer (TTA/ TTS decreased). However, the effect of $e H M I$ was dependent on the vehicle behavior. For Gentle and Early braking, the eHMI had a statistically significant effect with a high effect size. Conversely, the eHMI did not have a statistically significant effect holistically over the course of the measurement points for Aggressive braking and Constant speed conditions, and showed a medium and a low effect size 


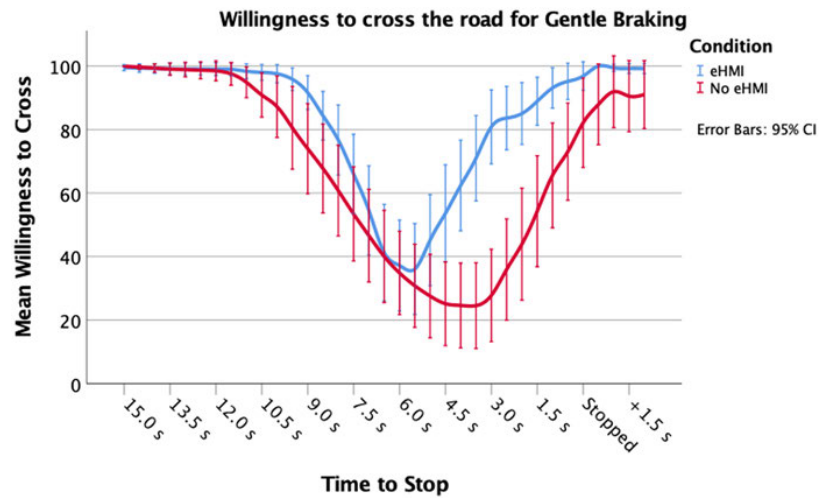

Figure 3: Gentle braking.

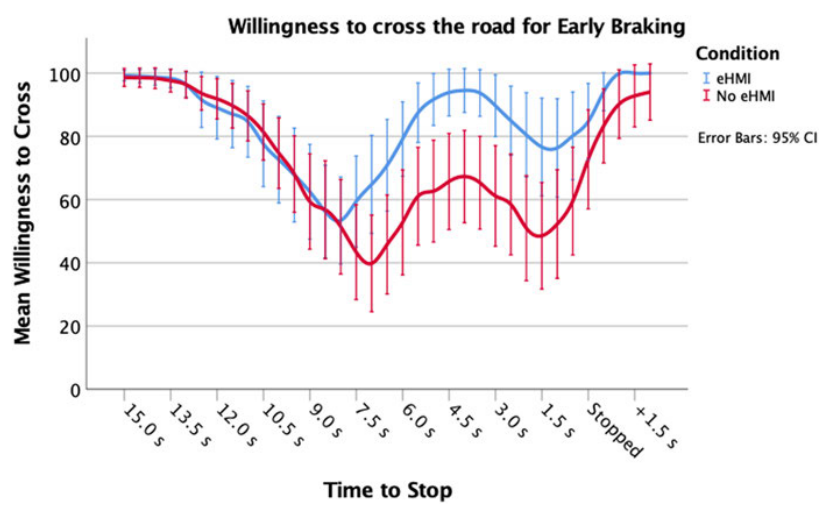

Figure 4: Early braking.

respectively. Similarly, the interaction effect Time ${ }^{*} e H M I$ varied - it was statistically significant for Gentle and Early

Table 2: Test statistics of the effects of time, eHMI, and their interaction on pedestrians' willingness to cross.

\begin{tabular}{lrrr}
\hline Condition & F & Sig. & Effect size (r) \\
\hline Time & & & \\
\hline Gentle braking & 47.19 & $<0.001$ & 0.82 \\
Early braking & 11.04 & $<0.001$ & 0.57 \\
Aggressive braking & 58.29 & $<0.001$ & 0.85 \\
Constant speed & 153.52 & $<0.001$ & 0.94 \\
\hline eHMI & & & \\
\hline Gentle braking & 18.79 & $<0.001$ & 0.67 \\
Early braking & 13.65 & 0.001 & 0.61 \\
Aggressive braking & 2.28 & 0.146 & 0.31 \\
Constant speed & 0.035 & 0.852 & 0.04 \\
\hline Time ${ }^{*}$ eHMI & & & \\
\hline Gentle braking & 5.13 & $<0.001$ & 0.43 \\
Early braking & 3.269 & $<0.001$ & 0.35 \\
Aggressive braking & 1.247 & 0.187 & 0.23 \\
Constant speed & 0.473 & 0.989 & 0.15 \\
\hline
\end{tabular}

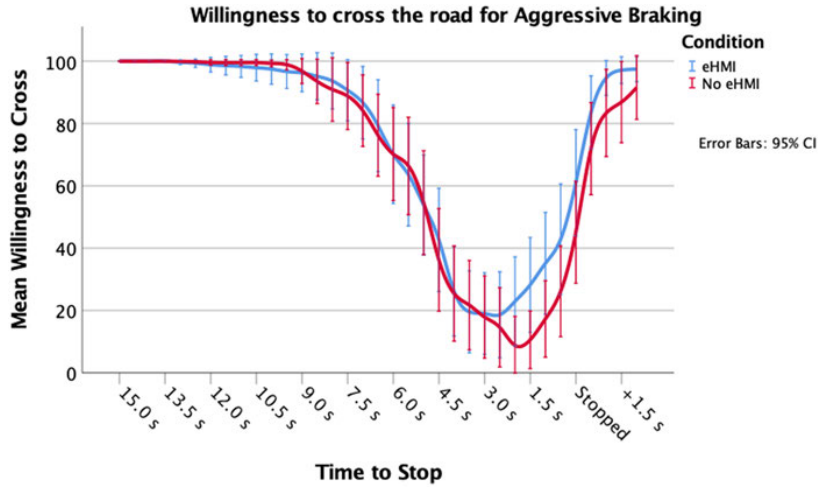

Figure 5: Aggressive braking.

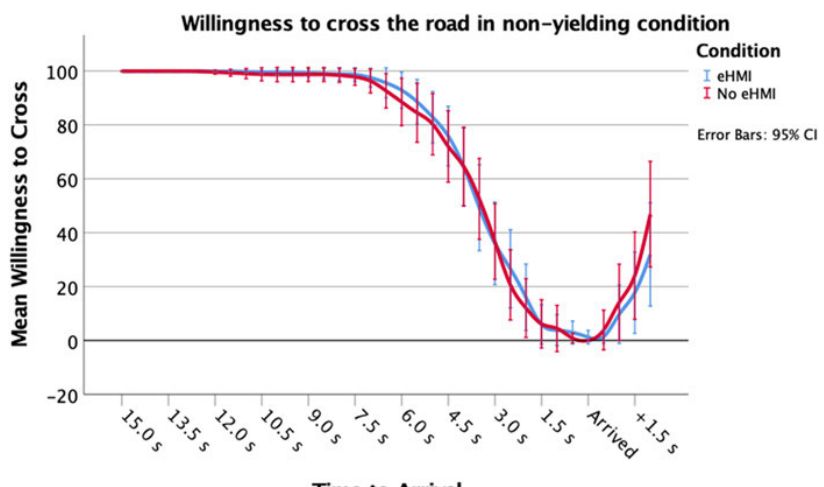

Figure 6: Constant speed.

braking, and not significant for Aggressive braking and Constant speed.

To investigate whether the eHMI had a significant effect at any specific TTA/TTS points in addition to its holistic effect across the entire experience, we conducted a ttest on the eHMI and No-eHMI conditions for every TTA/ TTS measurement point. The test statistics and the effect sizes are shown in table 3.

Results show that for gentle and early braking behaviors, the eHMI had a significant effect for a stretch of time as the AV approached the pedestrian. The willingness to cross for the $e H M I$ condition is significantly higher than for the No-eHMI condition at several TTS measurement moments. For Gentle braking, the eHMI has a statistically significant, medium-size effect, increasing pedestrians willing to cross, between TTS of $3.5 \mathrm{~s}$ and $1.5 \mathrm{~s}$. For the Early braking condition, the eHMI had a statistically significant, medium to high effect, increasing pedestrians' willingness to cross, between TTS of $5.5 \mathrm{~s}$ and $2.0 \mathrm{~s}$. In Figures 3 and 4, it can be seen that the difference between eHMI and No eHMI already begins at $-7 \mathrm{~s}$ for Gentle braking and $-8 \mathrm{~s}$ for Early braking, but these differences are not significant be- 
Table 3: Main effects of the presence of eHMI for different Time-to-stop (TTS - when the vehicle stopped) or Time-to-arrival (TTA - when the vehicle drove by) measuring points for different driving behaviors. The corresponding effect size (Cohen's d) are reported and the TTS/TTA points where the eHMI had a significant effect at a Bonferroni-corrected confidence level of 0.002 are highlighted in bold and italics.

\begin{tabular}{|c|c|c|c|c|c|c|c|c|c|c|c|c|}
\hline \multirow[t]{2}{*}{ TTA/ TTS (s) } & \multicolumn{3}{|c|}{ Gentle braking } & \multicolumn{3}{|c|}{ Early braking } & \multicolumn{3}{|c|}{ Aggressive braking } & \multicolumn{3}{|c|}{ Constant speed } \\
\hline & $t$ & $\mathbf{p}$ & $\bar{d}$ & $t$ & $\mathbf{p}$ & $\bar{d}$ & $t$ & $\mathbf{p}$ & $\bar{d}$ & $t$ & $\mathbf{p}$ & c \\
\hline-11.0 & -1.972 & 0.061 & 0.386 & 0.334 & 0.742 & 0.080 & 1.000 & 0.328 & 0.204 & -1.000 & 0.328 & 0.222 \\
\hline-10.5 & -2.364 & 0.027 & 0.577 & 0.546 & 0.590 & 0.137 & 1.014 & 0.321 & 0.224 & -1.000 & 0.328 & 0.204 \\
\hline-10.0 & -2.300 & 0.031 & 0.613 & 0.297 & 0.769 & 0.070 & 0.951 & 0.352 & 0.221 & -1.000 & 0.328 & 0.179 \\
\hline-9.5 & -2.420 & 0.024 & 0.678 & 0.041 & 0.968 & 0.010 & 1.001 & 0.328 & 0.657 & -1.000 & 0.328 & 0.157 \\
\hline-9.0 & -2.453 & 0.022 & 0.696 & -0.362 & 0.720 & 0.087 & 0.150 & 0.882 & 0.022 & -1.000 & 0.328 & 0.116 \\
\hline-8.5 & -2.177 & 0.040 & 0.622 & 0.078 & 0.938 & 0.016 & -0.682 & 0.502 & 0.110 & -1.000 & 0.328 & 0.068 \\
\hline-8.0 & -1.947 & 0.064 & 0.527 & -0.293 & 0.772 & 0.059 & -0.701 & 0.491 & 0.138 & -1.417 & 0.171 & 0.074 \\
\hline-7.5 & -1.769 & 0.09 & 0.387 & -1.759 & 0.092 & 0.460 & -0.542 & 0.593 & 0.088 & -1.082 & 0.291 & 0.132 \\
\hline-7.0 & -1.168 & 0.255 & 0.233 & -2.505 & 0.020 & 0.686 & -0.630 & 0.535 & 0.504 & -0.697 & 0.493 & 0.135 \\
\hline-6.5 & -0.135 & 0.894 & 0.034 & -2.528 & 0.019 & 0.701 & -0.574 & 0.572 & 0.125 & -0.997 & 0.330 & 0.227 \\
\hline-6.0 & -0.267 & 0.792 & 0.072 & -3.048 & 0.006 & 0.775 & -0.074 & 0.942 & 0.018 & -1.035 & 0.312 & 0.246 \\
\hline-5.5 & -0.521 & 0.608 & 0.162 & -3.739 & 0.001 & 0.871 & 0.235 & 0.817 & 0.055 & -0.884 & 0.386 & 0.192 \\
\hline-5.0 & -1.714 & 0.100 & 0.540 & -3.903 & 0.001 & 0.958 & 0.020 & 0.984 & 0.006 & -0.512 & 0.614 & 0.123 \\
\hline-4.5 & -2.645 & 0.014 & 0.840 & -3.838 & 0.001 & 0.993 & -0.900 & 0.378 & 0.190 & -0.488 & 0.631 & 0.136 \\
\hline-4.0 & -3.498 & 0.002 & 1.155 & -3.759 & 0.001 & 1.007 & -0.219 & 0.829 & 0.052 & 0.002 & 0.998 & 0.001 \\
\hline-3.5 & -4.268 & $<0.001$ & 1.457 & -3.674 & 0.001 & 1.033 & 0.129 & 0.898 & 0.035 & 0.290 & 0.775 & 0.078 \\
\hline-3.0 & -5.535 & $<0.001$ & 1.702 & -3.165 & 0.004 & 0.913 & -0.205 & 0.839 & 0.061 & 0.015 & 0.988 & 0.004 \\
\hline-2.5 & -5.290 & $<0.001$ & 1.514 & -3.095 & 0.005 & 0.819 & -0.492 & 0.627 & 0.148 & -0.851 & 0.404 & 0.201 \\
\hline-2.0 & -4.346 & $<0.001$ & 1.221 & -3.609 & 0.001 & 0.828 & -1.768 & 0.091 & 0.514 & -0.702 & 0.490 & 0.165 \\
\hline-1.5 & -3.924 & 0.001 & 1.085 & -3.243 & 0.004 & 0.734 & -2.310 & 0.031 & 0.588 & 0.085 & 0.933 & 0.026 \\
\hline-1.0 & -3.600 & 0.002 & 0.929 & -2.458 & 0.022 & 0.621 & -2.318 & 0.030 & 0.506 & 0.156 & 0.878 & 0.047 \\
\hline-0.5 & -3.075 & 0.005 & 0.809 & -2.068 & 0.050 & 0.560 & -2.179 & 0.040 & 0.420 & -0.903 & 0.376 & 0.272 \\
\hline 0.0 & -2.017 & 0.056 & 0.594 & -1.210 & 0.239 & 0.361 & -1.648 & 0.114 & 0.386 & -1.000 & 0.328 & 0.295 \\
\hline+0.5 & -1.966 & 0.062 & 0.567 & -1.646 & 0.113 & 0.491 & -1.486 & 0.152 & 0.375 & 0.623 & 0.539 & 0.187 \\
\hline+1.0 & -1.366 & 0.019 & 0.398 & -1.838 & 0.079 & 0.531 & -1.734 & 0.097 & 0.470 & 0.606 & 0.551 & 0.145 \\
\hline+1.5 & -1.668 & 0.109 & 0.459 & -1.519 & 0.142 & 0.439 & -1.574 & 0.130 & 0.464 & 0.459 & 0.651 & 0.122 \\
\hline+2.0 & -1.682 & 0.106 & 0.452 & -1.390 & 0.178 & 0.401 & -1.153 & 0.261 & 0.336 & 1.151 & 0.262 & 0.280 \\
\hline
\end{tabular}

cause the Bonferroni adjustment makes the test very conservative. Overall, this indicates that in these TTS measurements, the eHMI helps pedestrians to comprehend the vehicle's yielding intentions more clearly. In contrast, for Aggressive braking and Constant speed, the effect of the eHMI was not statistically significant at any TTS/ TTA measuring point, and the effect sizes were small.

\subsection{Gaze behavior}

Of the 26 participants the study was conducted with, data from seven participants suffered from poor gaze sampling during the interaction moments and more than $40 \%$ of the gaze samples were lost due to technical difficulties. We excluded these from the gaze behavior analysis. Data from the rest of the 19 participants recorded a gaze sampling rate of over $80 \%$. We coded the eye tracking data for the rest of the 19 participants using the Tobii Pro Lab ${ }^{3}$

$3 \mathrm{https} / / /$ www.tobiipro.com/product-listing/tobii-pro-lab/, last access 2020-07-09. software. In order to categorize the gaze behaviors of the pedestrians, eight Areas of Interest (AOI) were defined on the car (see Figure 7). For each behavior with and without

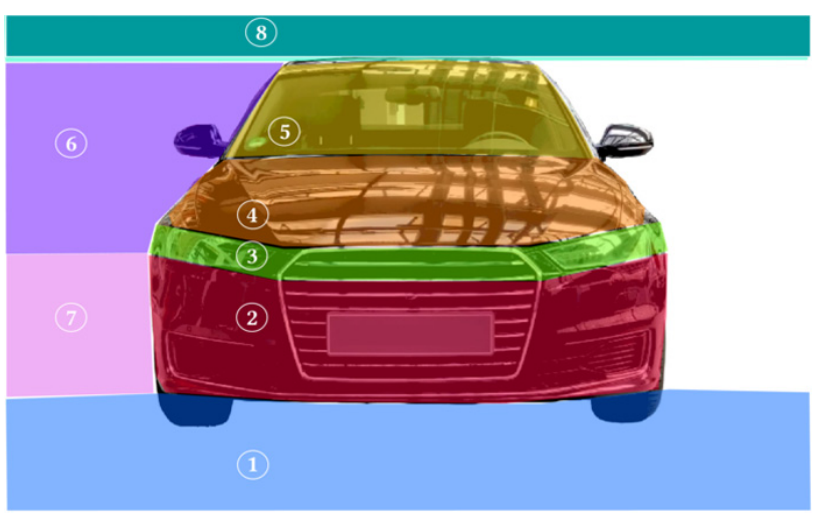

Figure 7: The Areas of Interest (AOIs) defined on the vehicle: (1) Road surface in front of the AV; (2) Bumper; (3) eHMI [when eHMI is present], headlights and grill [when eHMI is absent]; (4) Hood; (5) Windshield; (6) Side; (7) Wheels; (8) Environment. 


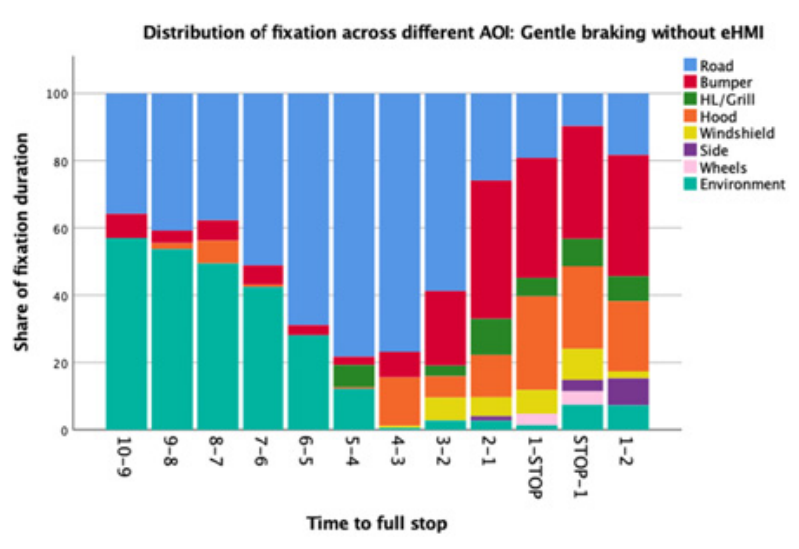

(a) No eHMI

Figure 8: Gentle braking.

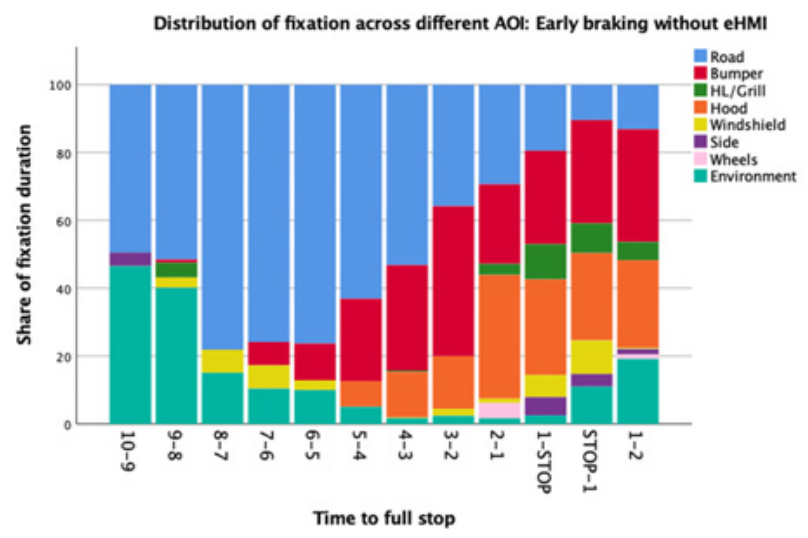

(a) No eHMI

Figure 9: Early braking.

eHMI, the fixations were manually coded by mapping a fixation to the corresponding AOI.

We asked participants to respond as they normally would in the presence of the vehicle in order to preserve ecological validity. They were not advised against turning their heads or looking around, and they were not asked to specifically look only in the direction of the approaching vehicle. In the process of the study, in some cases, participants turned their heads and looked around at their surroundings, which led to the car being momentarily hidden from view. Such data were coded as fixations on the environment (AOI 8). The start of the mapping of gaze behavior on different AOIs of the car was executed from a TTA/ TTS of $10 \mathrm{~s}$ away from the pedestrian until up to $2 \mathrm{~s}$ after stopping/ arrival, and gaze samples were pooled in one second intervals (Time of Interest, or TOI intervals were $1 \mathrm{~s}$ ) corresponding to the intervals of Time-to-Stop (TTS) or Time-

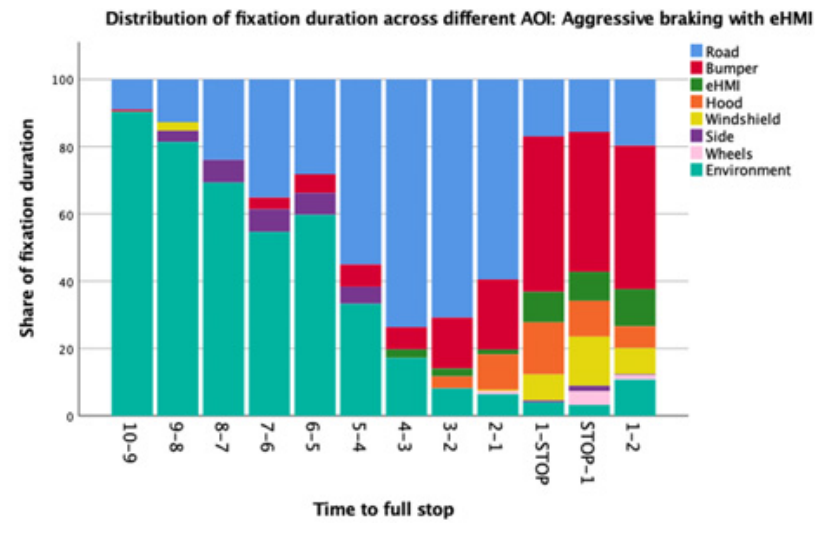

(b) eHMI

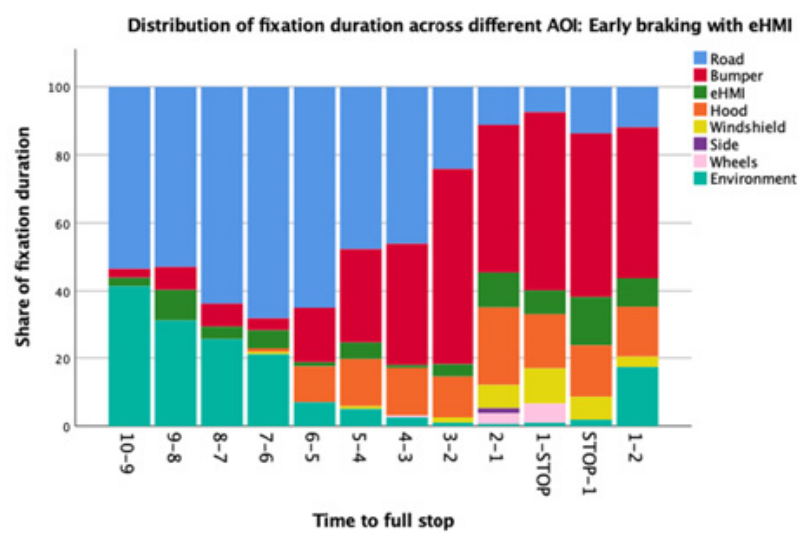

(b) eHMI
to-Arrival (TTA), and the dependent variable analyzed was the fixation duration across different AOIs within these TOI intervals.

The heat maps in Appendix A show the pattern of gaze behavior of the pedestrians in response to the approaching vehicle. Figures 8 to 11 show the distribution of the fixation durations across various AOIs at the analyzed time intervals. The data from these graphs show that for all yielding behaviors, pedestrians look largely at the road surface in front of the vehicle or the environment, until the approaching AV is relatively close to the pedestrian. Closer to the stopping point, pedestrians are observed to look more at the bumper, headlight/ grill/ eHMI, hood, and windshield areas. In contrast, for the non yielding condition (constant speed), the pedestrians do not fixate on the car until the very last moments leading up to its arrival at the pedestrians' location. 


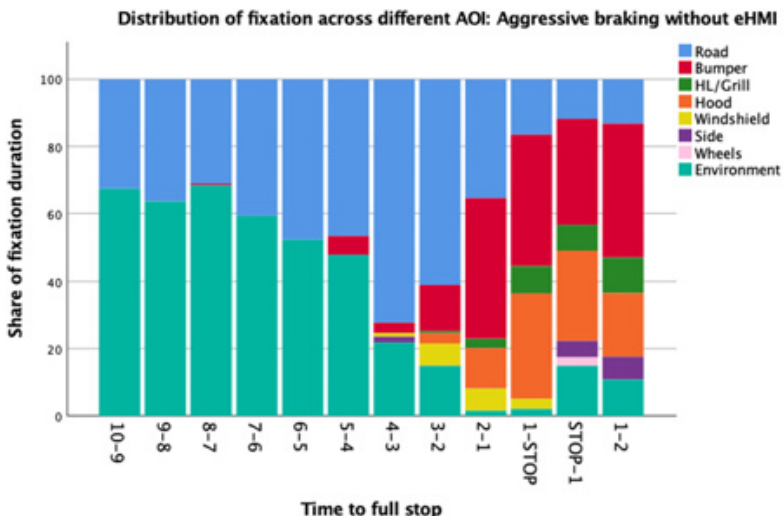

(a) No eHMI

Figure 10: Aggressive braking.

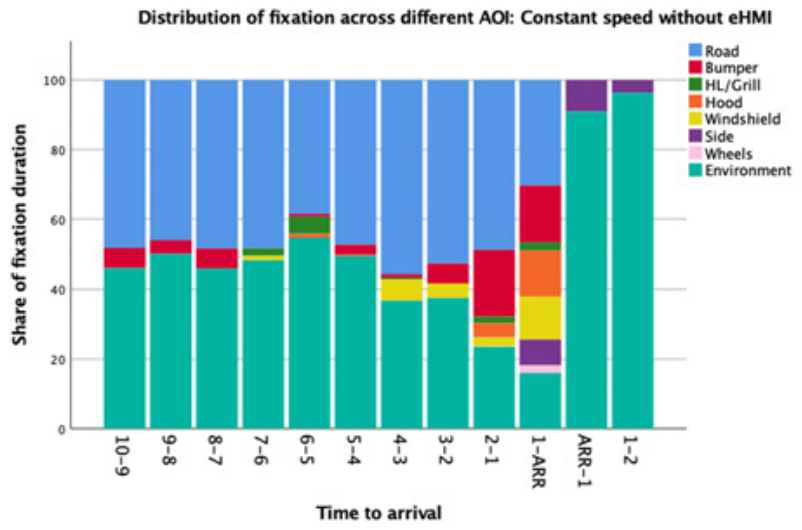

(a) No eHMI

Figure 11: Constant speed.

For each time interval in every behavior condition where the pedestrians were observed to fixate on the eHMI AOI (for the eHMI condition) and the headlight/ grill AOI (for the No-eHMI condition), we conducted a t-test to investigate whether the fixations on AOI number 3 (corresponding to the eHMI or the headlights/ grill) were different as a result of the eHMI activating. We found no statistically significant differences in the fixation durations in this area as a result of the eHMI at any time interval for any behavior condition, and the effect sizes were small.

\subsection{Qualitative feedback}

In addition, we collected qualitative feedback through semi-structured interviews at the end of the experiment to gain subjective insights from our participants. Although the quantitative data was obtained from 24 participants (as

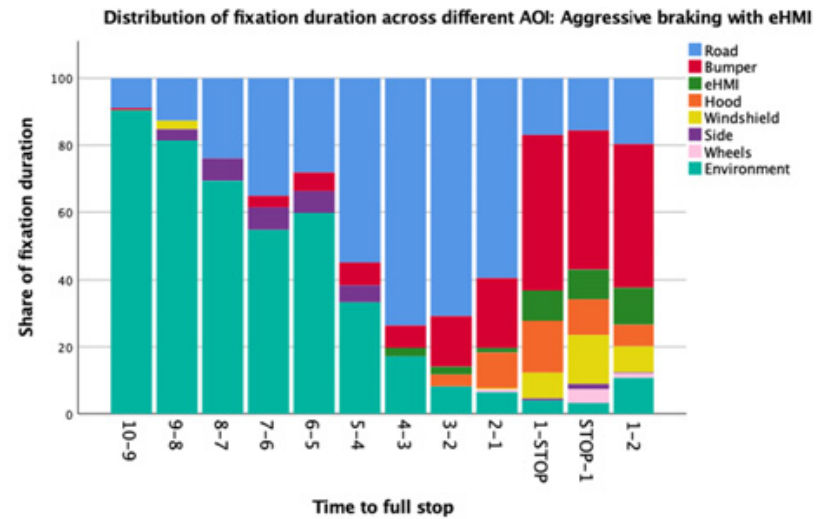

(b) eHMI

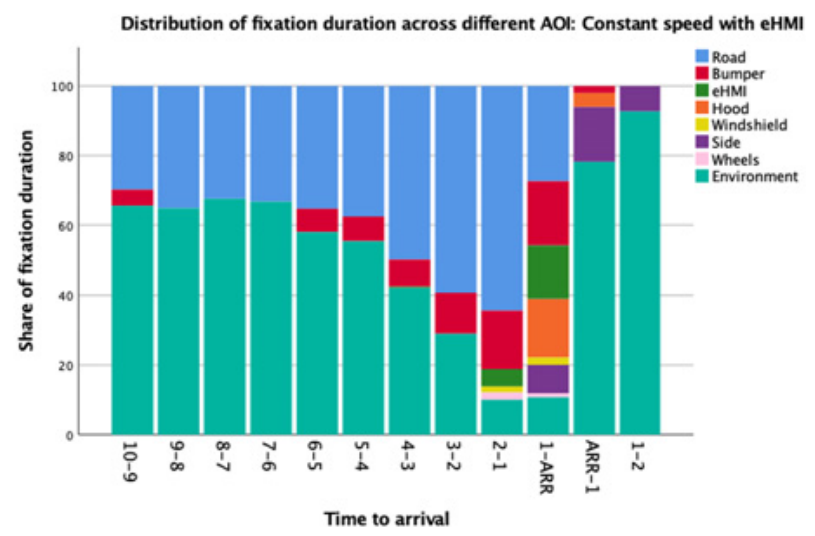

(b) eHMI a result of lost data from two participants), each of the 26 participants involved in the study provided their qualitative feedback. This section outlines insights from the thematic analysis applied to the qualitative data, furnished with selected participant quotes.

\subsubsection{Pedestrians feel more confident about their crossing decisions in the presence of an eHMI}

Participants commented that in general they felt more confident in the vehicle's yielding intentions when the eHMI was present. With the eHMI, participants mentioned feeling more at ease, confident, and willing to cross the road (P4, P8, P15), more certain about the vehicle's intentions (P11), and considered it "additional help" (P13, P16). Others mentioned actively looking for the light to make 
their decision (P6, P23) and reported that they trusted the light to an extent that they felt it missing when it was not there (P24). However, a participant also remarked that while the eHMI helped, it "wasn't a $100 \%$ confirmation" for them to be willing to cross and that they depended on the vehicle's speed (P16).

\subsubsection{Preferences differ between braking behaviors}

Opinions were split regarding the pedestrians' preference for the ideal braking behavior. Every participant mentioned that they liked the Gentle braking behavior, and some clarified that this was because for them it corresponded most closely with how a human driver would respond to a pedestrian waiting to cross (P16, P20-P22).

However, people disagreed on their perception of the Early braking behavior. Two participants mentioned that earlier braking made the vehicle's yielding intention clear to them in advance and they felt more confident in their crossing decision (P12, P25). However, most participants (16/26) found this behavior confusing, and some specifically mentioned disliking this, even worse than the $\mathrm{Ag}$ gressive braking behavior (P17, P22). For them, the fact that the vehicle braked and slowed down earlier, but still kept approaching them caused doubt and mistrust in the vehicle's intentions. Participants generally responded with an intense reaction to the Early braking behavior - either strongly liking it or strongly disliking it.

On the whole, based on their response to the Early braking behavior, participants' response to the Aggressive braking behavior either came second or last among the three yielding behaviors, and in general, was not a preferred behavior. Participants mentioned that even if the eHMI was active and showed the yielding message for this behavior, they felt unsafe to cross as the speed of the vehicle was too high (P16, P20, P23). One participant also mentioned that when the eHMI showed the yielding message despite the AV approaching quite fast until the last moment before braking aggressively, they "felt betrayed" (P8).

\subsection{Evaluation of hypotheses}

From these data, we are able to determine the validity of our hypotheses as follows:

H1: For a yielding vehicle, pedestrians' willingness to cross will be higher when the AV is equipped with an eHMI than without an eHMI. Insights from our study show that an eHMI has the potential to mitigate ambiguity and resolve confusion regarding an AV's yielding intention when the speed of the AV is low. However, an eHMI does not unilaterally increase pedestrians' willingness to cross for a yielding vehicle. Especially when the speed of the vehicle is high or the AV brakes aggressively, pedestrians fall back on the vehicle behavior as the primary source of information about its yielding intent. This leads us to partially accept H1 - the eHMI had an effect on pedestrians' crossing behaviors in certain conditions.

H2: If an eHMI message contradicts the behavior of the $A V$, pedestrians will fall back on the vehicle behavior based cues. Results also show that when the behavior of the vehicle contradicts the eHMI's yielding message (e. g. in the Aggressive braking behavior), pedestrians fall back on the behavioral cues and do not blindly trust the eHMI. Even though the eHMI showed that the AV intended to yield, the high speed of the vehicle did not inspire confidence in the eHMI's message and the pedestrians waited until the AV slowed down. This leads us to accept $\mathrm{H} 2$ - our data and analyses support the prediction that pedestrians rely on vehicle kinematics.

H3: when an eHMI is on, pedestrians will fixate on it longer than the corresponding location of the $A V$ when the eHMI is off. Analysis of fixation data show that pedestrians did not fixate on the eHMI when it was active significantly more than when it was inactive, and the effect size of eHMI on fixation duration was small. This leads us to reject H3 there is no evidence that a light-based eHMI affects pedestrians' attention significantly.

\section{Discussion}

Our results show that eHMIs can play a role in mitigating pedestrians' ambiguity in understanding an AV's intentions in yielding situations, and can help pedestrians to make quicker decisions. However, the behavior of the vehicle has an impact in this. We reflect on the findings of our study and discuss the implications on the design of an effective AV-pedestrian communication paradigm.

\subsection{The effect of eHMI}

Subjective feedback from the interviews after the experiment revealed that pedestrians felt the eHMI had a positive effect overall in their decision making process and improving the experience of interaction. Quantitative results show that the effect of the eHMI was significant primarily in the Gentle braking and Early braking conditions. The 

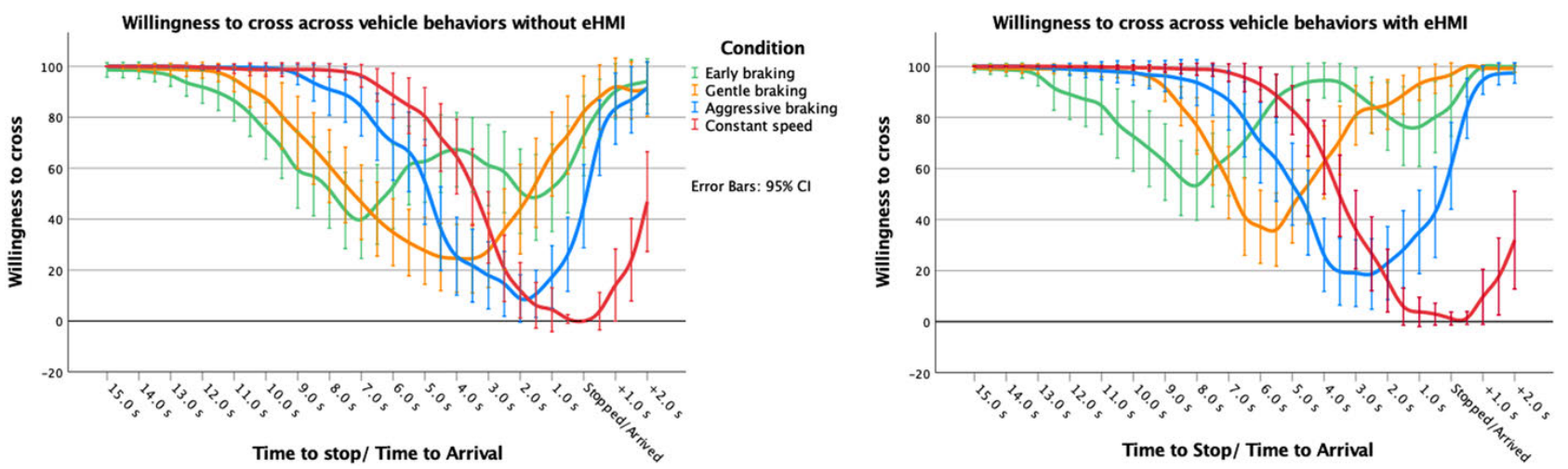

Figure 12: Pedestrians' willingness to cross the road across different vehicle behaviors. Left: No eHMI; Right: eHMI.

impact of the eHMI was statistically significant for only a small period of the TTS measures for Aggressive braking and none of the measures of TTA for Constant speed conditions, and for these behaviors, the effect size was not high. The finding that eHMI is generally effective is in line with previous findings $[1,4,13,14,16]$. However, this comes with the caveat - the effectiveness of the eHMI is contingent on the behavior and the speed of the vehicle. An additional point to consider is that in this study, the functionality of the eHMI was explained to the participant. In a naturalistic setting, the actual effectiveness of an eHMI will also depend on the intuitiveness of the deployed concept.

\subsection{Driving behavior and eHMI}

Results show that the driving behavior of the vehicle exhibited by its movement patterns had an impact on the efficacy of the eHMI in terms of improving pedestrians' willingness to cross. Pedestrians did not simply take the message of the eHMI as the ultimate truth and verified whether the behavior of the vehicle corresponded with the eHMI communication.

This is particularly seen in the Aggressive braking condition: even though the eHMI activated the 'yielding' message at $45 \mathrm{~m}$ from the pedestrian, the car was still driving at $50 \mathrm{~km} / \mathrm{h}$ at this point. The car braked hard at around $19 \mathrm{~m}$ from the pedestrian, at which point the eHMI was already displaying the yielding message for some time. However, the pedestrians' willingness did not rise as a result of the message of the eHMI alone. This condition was an example of when the message of the eHMI and the behavior of the vehicle did not correspond to each other until the last moment of the vehicle coming to a complete stop.

Similarly, for the early braking behavior, fig. 4 shows an interesting pattern. The willingness to cross drops as the car approaches and rises as it brakes initially and slows down, as expected. However, the willingness to cross drops again as the AV approaches closer until it slows - pedestrians assumed with the initial braking that the AV was going to yield, but could no longer be sure when it kept approaching, until it slowed down to a complete stop. The presence of the eHMI mitigated the ambiguity to a certain extent, but did not resolve it entirely. The pattern of willingness to cross for the eHMI condition mirrors that of the No-eHMI condition.

Results showed that the effect of the eHMI is dependent on the vehicle's behavior. Figure 12 shows that in general, the pattern of pedestrians' willingness to cross for the different behaviors remains consistent across the two eHMI conditions. Although the eHMI can reduce the degree of ambiguity to a certain extent, the general shape of the graphs for the different behavior conditions remains consistent across the two eHMI conditions. This indicates that the presence of the eHMI did not unilaterally convince the pedestrians that the vehicle was going to yield in any condition, and they still based their judgment and decision on the vehicle's driving behavior. When the speed of the vehicle is high, the eHMI had little to no effect in modulating pedestrians' willingness to cross. Only when the speed of the vehicle is low enough that it could mean yielding intention, but still high enough to be a threat, did the eHMI have an effect by elucidating the AV's intention. This indicates that when the eHMI's yielding message and the vehicle's behavior contradict each other, people still give more priority to the vehicle's behavior. Pedestrians do not blindly follow instructions from an eHMI. This finding contradicts previous research [15] which found that people tend to start out with a high degree of trust for eHMIs and are not hesitant to follow the message of an eHMI.

In contrast, previous research also claimed that the vehicle's movement patterns are enough for expressing the 
intentions of a vehicle and that further communications may not be necessary $[10,19,20]$. Our results show that in specific situations (Gentle and Early braking conditions), when the speed of the AV was low enough, the eHMI did have a positive effect on pedestrians' willingness to cross. Other prior work has also investigated a situation when an AV slowed down without stopping [7]. It showed that an eHMI was able to clarify the AV's intention to not yield even when the slowing behavior of the vehicle would seem to indicate that it would stop. We posit that while vehicle movement patterns may be enough for many situations, an eHMI may still have the potential to clarify the intention of the vehicle and reduce ambiguity, particularly in lowspeed situations when the stopping intention of the $\mathrm{AV}$ is unclear.

Besides, there is an added consideration for the breaching behaviors (early and aggressive breaking) when the message of the vehicle and its behavior appeared to go against each other. Although the behavior of the vehicle never entirely contradicted the eHMI (i. e. there was no situation where the car did not stop when the eHMI said it would), the apparent misalignment of the vehicle behavior and communication may be interpreted as unsafe behavior, which could damage the trust on the vehicle.

\subsection{Ideal yielding behavior}

As expected, Aggressive braking was not appreciated by any of the participants. However, opinion was split between Gentle braking and Early braking. Many participants found the initial pitching due to the first braking moment in the early braking condition to be a helpful and obvious indication that the AV was slowing down. In the Gentle braking condition, the slowing of the car was not comparably obvious, and took more time. Others indicated discomfort with the Early braking condition since the car continued to approach after braking - they could not be certain that the AV was indeed stopping for them. Previous research showed that a defensive deceleration with a stronger vehicle pitch due to hard initial braking reduces the time it takes pedestrians to understand an approaching vehicle's yielding intention [12]. However, our findings contradict this result. Besides, defensive and hard initial braking may also cause disruptions to traffic flow. We posit that a uniform, controlled, and gentle braking behavior in tandem with an eHMI elucidating the AV's intent is a good candidate for an effective and pleasant interaction between an $\mathrm{AV}$ and a pedestrian.

\subsection{Pedestrians' gaze patterns}

Previous research showed that a distinct pattern exists in the gaze behavior of pedestrians when interacting with an approaching manually-driven vehicle [11] - pedestrians looked at the road surface in front of the car or the environment when the car is at a distance, and progressively fixate on the bumper, headlights-and-grill, hood, and windshield areas as the car approaches closer. Our result shows that this pattern is also seen in interaction with an AV. The major difference was found in the fixation intensity on the windshield. Earlier research showed that pedestrians had a strong tendency to look at the windshield when the vehicle was close to them, and the authors speculated that this was likely because the pedestrians sought confirmatory information about the driver's intent. However, such a strong tendency to look at the windshield was not found with the AV, both with and without the eHMI. We conjecture that since the pedestrians believed that they were interacting with an $\mathrm{AV}$ with no human driver controlling the vehicle, the need to look inside the vehicle near the windshield area was no longer there.

Furthermore, analysis showed that pedestrians' fixations on the eHMI were not significantly higher than their fixation on the corresponding headlights-and-grill area of the AV when the eHMI was switched off. An eHMI concept based on user-centered design principles of providing the right information and the right place and time should also present information where it is expected. The fixation distribution shows that as the AV approached and pedestrians looked at the vehicle, most of the fixations occurred on the bumper and hood. One may argue that placing a light-based emissive eHMI will attract attention by increasing the perceptual salience irrespective of where it is placed. However, our results indicate that the presence of the eHMI did not significantly alter the gaze behavior - pedestrians did not look at the corresponding areas more when the eHMI was on. A consideration is that by the design of this experiment, the participants knew that sometimes they would be seeing the eHMI, and other times they would not. So it might be possible that even when the eHMI was off, they were looking at the headlights-and-grill area to see if the eHMI showed anything even if it was off. This could account for the lack of a significant difference in fixation duration on the AOI when the eHMI was on. However, despite a lack of increased fixations on the location of an eHMI, the positive effect of the eHMI was observed in Gentle and Early braking conditions in terms of the pedestrians' willingness to cross. We conjecture that even though pedestrians did not necessarily fixate directly on the eHMI, the message of the eHMI was seen by them 
in their parafoveal vision, and was effective without being distracting.

\subsection{Limitations and future work}

In order to limit confounding factors, we conducted the experiment in a simplified scenario involving only one car and one pedestrian on a straight and empty road devoid of any other traffic. Our findings provide the first results regarding pedestrians' willingness to cross in the specific driving behaviors explored with and without an eHMI in such a baseline scenario. An AV can yield in a multitude of ways, and with an increase in the complexity of the traffic situation, the gaze pattern may be more varied. Future research needs to extend this work in different traffic situations and in more dynamic scenarios involving multiple cars and pedestrians.

Additionally, in our study, we asked participants to merely indicate their willingness to cross using an input device rather than actually crossing the road. It is possible that due to a lack of potential physical harm, participants exhibited a more risk-taking behavior. However, we chose this setup to ensure a high level of control in the environment, ensure the participants' safety, and obtain continuous data on the variation in their willingness to cross as the vehicle approached.

We conducted this study with 4 distinctly different driving behaviors and showed that the effectiveness of the eHMI is dependent on the behavior. However, a vehicle can exhibit a range of different driving behaviors that vary in nuanced and subtle ways. Different driving behaviors of a vehicle result in a complex interplay between its speed and distance from the pedestrian, and influence pedestrians' decisions. Although we showed that eHMIs tend to be more effective for certain driving behaviors, constructing a model of how speed and distance impact the effectiveness of an eHMI was out of the scope of this study, and needs to be addressed in future research.

\section{Conclusion}

This paper presents a controlled outdoor experiment that explored the effectiveness of explicit communication of vehicle intent through an eHMI in AV-pedestrian interaction situations, and the impact of different braking behaviors when the vehicle yields. Our results show that the answer to the question "Does an eHMI work?" is not straightforward, and is dependent on a complex interplay of the vehicle's speed and distance, which is a byproduct of the vehicle's driving behavior. In low-speed situations when the intention of the vehicle is unclear, explicit communication through an eHMI can resolve ambiguity about an AV's yielding intention and increase pedestrians' willingness to cross, thereby facilitating better traffic flow. The effect of an eHMI is less pronounced at higher speeds, when the behavioral cues of the vehicle take precedence. Pedestrians do not blindly trust a yielding message from an eHMI when the behavior of the vehicle does not concur with the message. Our empirical findings provide critical insight into the behavior of pedestrians in interactions with automated vehicles with eHMIs and add nuanced evidence to help drive the development of an effective communication paradigm for AV-pedestrian interaction.

Acknowledgment: We are greatly indebted to HELLA GmbH \& Co. KGaA, division Innovation Lighting and Marc Kaup for their collaboration and involvement in the design and execution of this study.

Funding: This research is supported by the Dutch Domain Applied and Engineering Sciences, which is part of the Netherlands Organization for Scientific Research (NWO), and which is partly funded by the Ministry of Economic Affairs (project number 14896).

\section{Appendix A. Fixation heat maps}




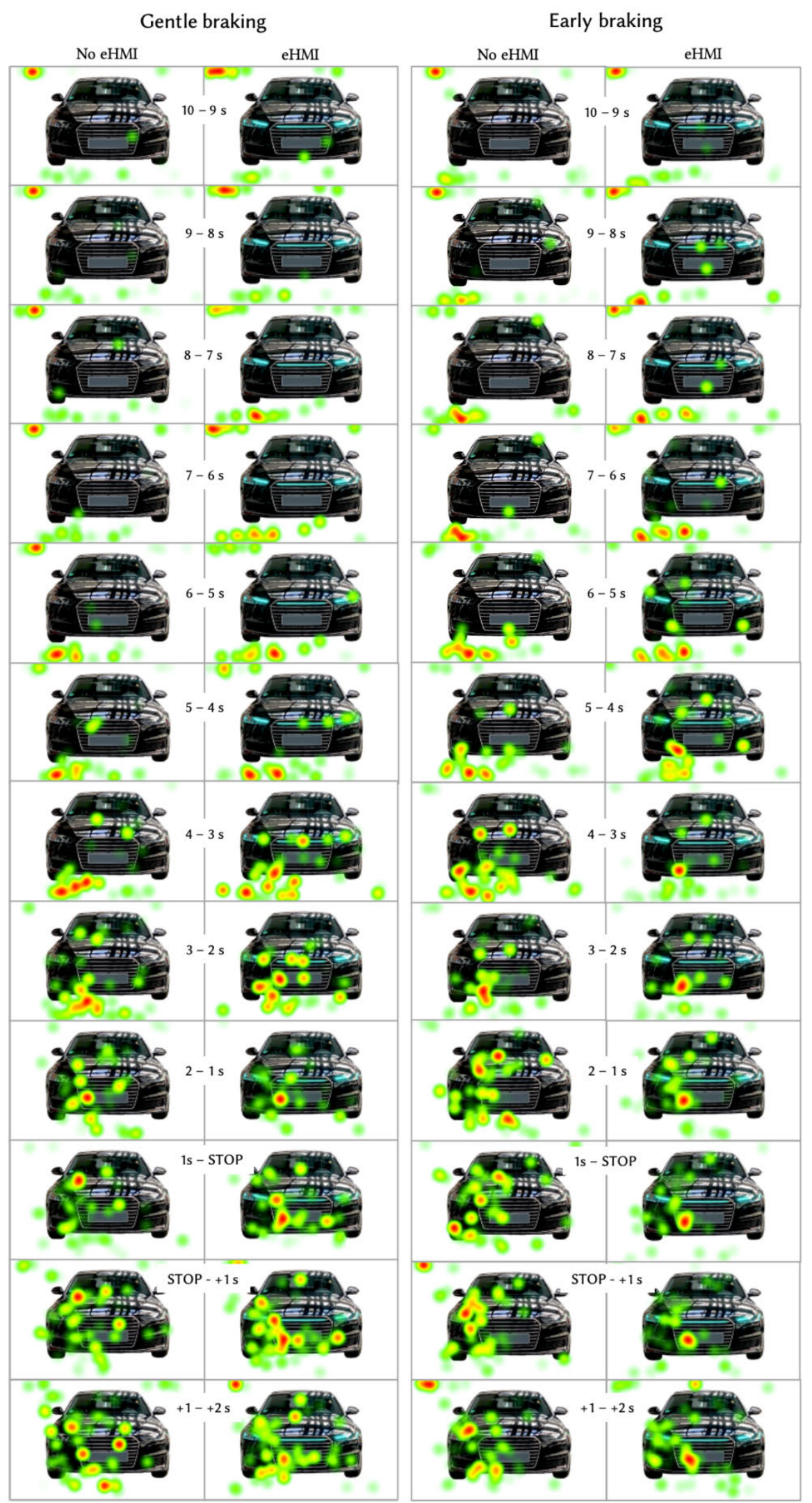

Figure 13: Heat maps of fixation duration through the approach of the AV across eHMI and No-eHMI conditions for Gentle braking and Early braking. To generate these heat maps, the Tobii I-VT Attention filter was used to visualize the absolute fixation duration data in $1 \mathrm{~s}$ intervals. 
Aggressive braking

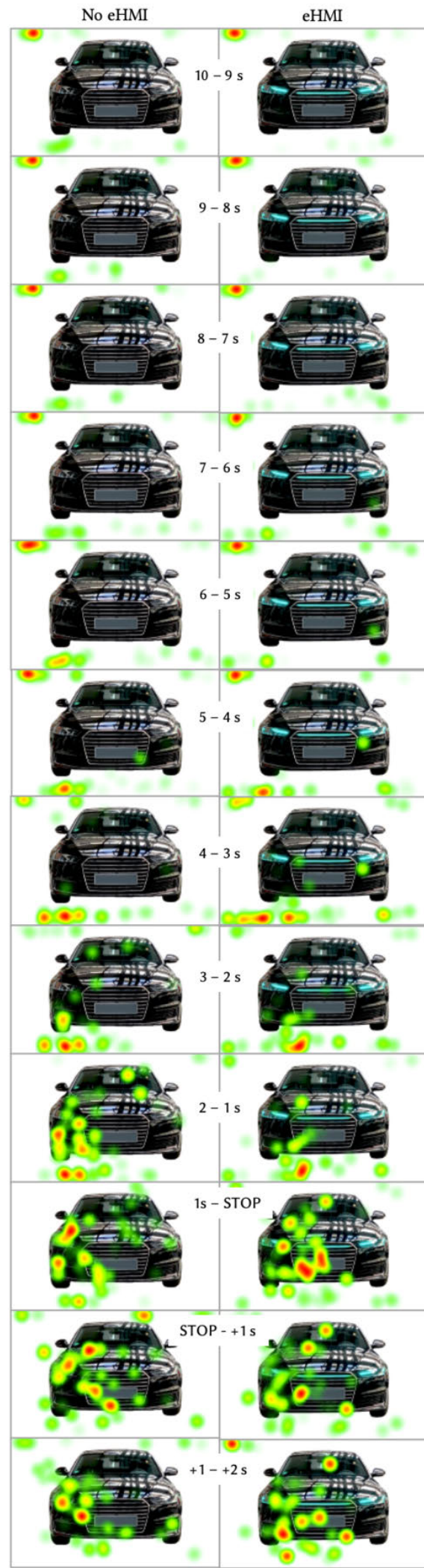

Constant speed

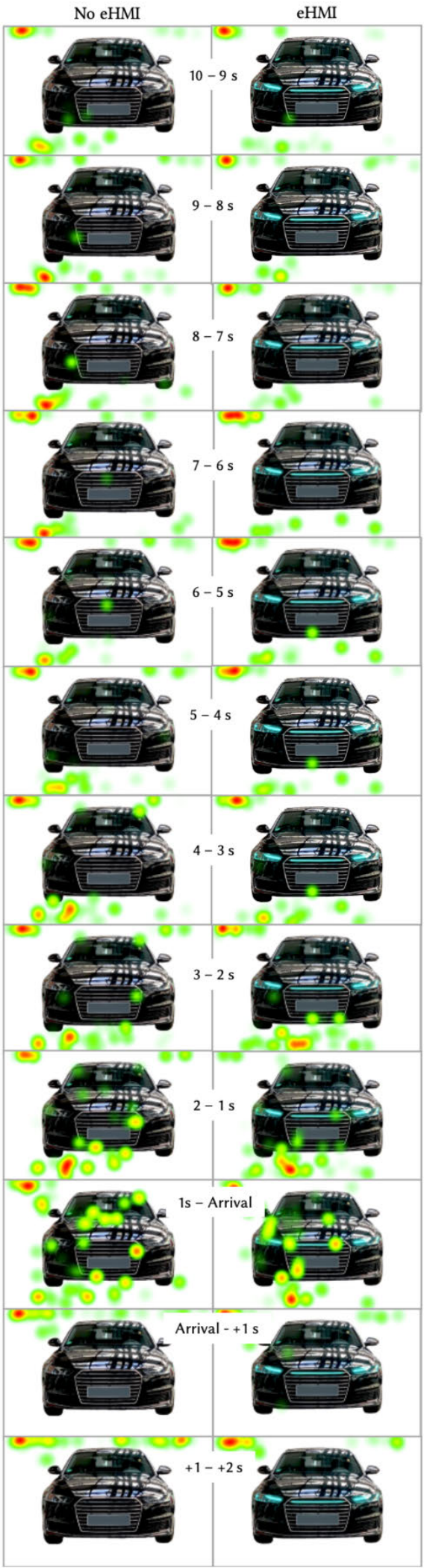

Figure 14: Heat maps of fixation duration through the approach of the AV across eHMI and No-eHMI conditions for Aggressive braking and Constant Speed. To generate these heat maps, the Tobii I-VT Attention filter was used to visualize the absolute fixation duration data in $1 \mathrm{~s}$ intervals. 


\section{Appendix B. Concept coding and evaluation parameters}

Table 4: Coding of the different eHMI dimensions for the concept used in this study as proposed in [6].

\begin{tabular}{|c|c|c|}
\hline$\#$ & eHMI dimension & Property \\
\hline 1 & Target road user & Pedestrian \\
\hline 2 & Vehicle type & Passenger car \\
\hline 3 & Modality of communication & Visual - abstract \\
\hline 4 & Color of eHMI & $\begin{array}{l}\text { Turquoise (bluish-green, } \\
\text { cyan) }\end{array}$ \\
\hline 5 & Covered states & $\begin{array}{l}\text { Cruising, Yielding, } \\
\text { Beginning to drive }\end{array}$ \\
\hline 6 & $\begin{array}{l}\text { Message of communication } \\
\text { in right-of-way negotiation }\end{array}$ & Intention announcement \\
\hline 7 & HMI placement & Headlights, grill \\
\hline 8 & Number of displays & $\begin{array}{l}1 \text { (light band spanning } \\
\text { headlights and grill) }\end{array}$ \\
\hline 9 & Number of messages & $\begin{array}{l}3 \text { (cruising, yielding, } \\
\text { beginning to drive) }\end{array}$ \\
\hline 10 & Communication strategy & Broadcast \\
\hline 11 & Communication resolution & Low \\
\hline 12 & $\begin{array}{l}\text { Multiple road user } \\
\text { addressing capability }\end{array}$ & Multiple/ Unlimited \\
\hline 13 & $\begin{array}{l}\text { Communication dependent } \\
\text { on distance/ time gap? }\end{array}$ & No \\
\hline 14 & Complexity to implement & $\mathrm{C} 1$ \\
\hline 15 & $\begin{array}{l}\text { Reliant on new vehicle } \\
\text { design? }\end{array}$ & No \\
\hline 16 & $\begin{array}{l}\text { Ability to show occupant } \\
\text { state/ shared control? }\end{array}$ & No \\
\hline 17 & $\begin{array}{l}\text { Ability to cater to VRUs with } \\
\text { accessibility needs/ hearing } \\
\text { impairment? }\end{array}$ & No \\
\hline 18 & Evaluation conducted? & Yes \\
\hline
\end{tabular}

\section{References}

1. S. Ackermans, D. Dey, P. Ruijten, R. H. Cuijpers, and B. Pfleging. The Effects of Explicit Intention Communication, Conspicuous Sensors, and Pedestrian Attitude in Interactions with Automated Vehicles. In CHI Conference on Human Factors in Computing Systems, pages 1-14, Honolulu, 2020.

2. C. G. Burns, L. Oliveira, V. Hung, P. Thomas, and S. Birrell. Pedestrian Attitudes to Shared-Space Interactions with Autonomous Vehicles - A Virtual Reality Study. In Advances in Intelligent Systems and Computing, volume 964, pages 307-316. Springer Verlag, 2020.

3. M. Clamann, M. Aubert, and M. L. Cummings. Evaluation of Vehicle-to-Pedestrian Communication Displays for Autonomous
Table 5: Summary of the different parameters of evaluation of the eHMI concept as recommended in [6].

\begin{tabular}{|c|c|c|}
\hline \# & Evaluation parameter & Condition \\
\hline 1 & Time of day & $\begin{array}{l}\text { Daytime - daylight } \\
\text { conditions }\end{array}$ \\
\hline 2 & $\begin{array}{l}\text { Number of simultaneous } \\
\text { road users }\end{array}$ & 1 \\
\hline 3 & $\begin{array}{l}\text { Number of simultaneous } \\
\text { vehicles }\end{array}$ & 1 \\
\hline 4 & $\begin{array}{l}\text { Methodology/medium of } \\
\text { testing }\end{array}$ & Controlled real-world \\
\hline 5 & Weather conditions & Clear skies, sunlight \\
\hline 6 & Road conditions & Clean roads \\
\hline 7 & Sample size & 24 \\
\hline 8 & Sample age & Mean $=26.21, S D=3.74$ \\
\hline 9 & Research methodology & $\begin{array}{l}\text { Mixed methods (quantitative } \\
+ \text { qualitative) }\end{array}$ \\
\hline
\end{tabular}

Vehicles. In 96th Annual Transportation Research Board Meeting, 2017.

4. K. de Clercq, A. Dietrich, J.P. Núñez Velasco, J. de Winter, and R. Happee. External Human-Machine Interfaces on Automated Vehicles: Effects on Pedestrian Crossing Decisions. Human Factors, 2019.

5. S. P. Deligianni, M. Quddus, A. Morris, A. Anvuur, and S. Reed. Analyzing and Modeling Drivers' Deceleration Behavior from Normal Driving. Transportation Research Record: Journal of the Transportation Research Board, 2663(1):134-141, Jan 2017.

6. D. Dey, A. Habibovic, A. Löcken, P. Wintersberger, B. Pfleging, A. Riener, M. Martens, and J. Terken. Taming the eHMI jungle: A classification taxonomy to guide, compare, and assess the design principles of automated vehicles' external human-machine interfaces. Transportation Research Interdisciplinary Perspectives, 7, 2020.

7. D. Dey, K. Holländer, M. Berger, B. Pfleging, M. Martens, and J. Terken. Distance-dependent eHMIs for the Interaction Between Automated Vehicles and Pedestrians. In AutomotiveUI (Accepted, 2020), pages 1-18, 2020.

8. D. Dey, M. Martens, B. Eggen, and J. Terken. Pedestrian road-crossing willingness as a function of vehicle automation, external appearance, and driving behaviour. Transportation Research Part F: Traffic Psychology and Behaviour, 65:191-205, Aug 2019.

9. D. Dey, M. Martens, C. Wang, F. Ros, and J. Terken. Interface Concepts for Intent Communication from Autonomous Vehicles to Vulnerable Road Users. In Adjunct Proceedings of the 10th International ACM Conference on Automotive User Interfaces and Interactive Vehicular Applications (AutomotiveUl'18), pages 82-86, 2018.

10. D. Dey and J. Terken. Pedestrian Interaction with Vehicles: Roles of Explicit and Implicit Communication. In AutomotiveUI'17 ACM 9th International Conference on Automotive User Interfaces and Interactive Vehicular Applications, Oldenburg, 2017.

11. D. Dey, F. Walker, M. Martens, and J. Terken. Gaze patterns in pedestrian interaction with vehicles: Towards effective design of external human-machine interfaces for automated 
vehicles. In Proceedings - 11th International ACM Conference on Automotive User Interfaces and Interactive Vehicular Applications, AutomotiveUI 2019, pages 369-378, 2019.

12. A. Dietrich, P. Maruhn, L. Schwarze, and K. Bengler. Implicit Communication of Automated Vehicles in Urban Scenarios: Effects of Pitch and Deceleration on Pedestrian Crossing Behavior. In Advances in Intelligent Systems and Computing, volume 1026, pages 176-181. Springer Verlag, Sept 2020.

13. A. Habibovic, V. M. Lundgren, J. Andersson, M. Klingegård, T. Lagström, A. Sirkka, J. Fagerlönn, C. Edgren, R. Fredriksson, S. Krupenia, D. Saluäär, and P. Larsson. Communicating Intent of Automated Vehicles to Pedestrians. Frontiers in Psychology, 9 (August), 2018.

14. K. Holländer, A. Colley, C. Mai, J. Häkkilä, F. Alt, and B. Pfleging. Investigating the influence of external car displays on pedestrians' crossing behavior in virtual reality. In Proceedings of the 21st International Conference on Human-Computer Interaction with Mobile Devices and Services, MobileHCI2019, ACM, New York, NY, USA, 2019.

15. K. Holländer, P. Wintersberger, and A. Butz. Overtrust in External Cues of Automated Vehicles: An Experimental Investigation. In 11th International Conference on Automotive User Interfaces and Interactive Vehicular Applications (AutomotiveUI'19), number April, pages 211-221, 2019.

16. L. Kooijman, R. Happee, and J.C. de Winter. How do eHMIs affect pedestrians' crossing behavior? A study using a head-mounted display combined with a motion suit. Information (Switzerland), 10(12):386, Dec 2019.

17. Y. Li, M. Dikmen, T. G. Hussein, Y. Wang, and C. Burns. To cross or not to cross: Urgency-based external warning displays on autonomous vehicles to improve pedestrian crossing safety. In Proceedings of the 10th International Conference on Automotive User Interfaces and Interactive Vehicular Applications, pages 188-197. ACM, 2018.

18. A. Löcken, C. Golling, and A. Riener. How should automated vehicles interact with pedestrians? A comparative analysis of interaction concepts in virtual reality. In Proceedings - 11th International ACM Conference on Automotive User Interfaces and Interactive Vehicular Applications, AutomotiveUI 2019, pages 262-274, 2019.

19. D. Moore, R. Currano, M. Shanks, and D. Sirkin. Defense Against the Dark Cars: Design Principles for Griefing of Autonomous Vehicles. In Proceedings of the 2020 ACM/IEEE International Conference on Human-Robot Interaction, pages 201-209, Association for Computing Machinery (ACM), New York, NY, USA, Mar 2020.

20. D. Moore, R. Currano, G. E. Strack, and D. Sirkin. The Case for Implicit External Human-Machine Interfaces for Autonomous Vehicles. In 11th International Conference on Automotive User Interfaces and Interactive Vehicular Applications (AutomotiveUl'19), pages 295-307, 2019.

21. S. Nordhoff, J. De Winter, M. Kyriakidis, B. Van Arem, and R. Happee. Acceptance of Driverless Vehicles: Results from a Large Cross-National Questionnaire Study. Journal of Advanced Transportation, 2018 (April), 2018.

22. S. Reig, S. Norman, C. G. Morales, S. Das, A. Steinfeld, and J. Forlizzi. A Field Study of Pedestrians and Autonomous
Vehicles. In Proceedings of the 10th International Conference on Automotive User Interfaces and Interactive Vehicular Applications - AutomotiveUl'18, pages 198-209, Toronto, Canada, 2018.

23. D. Rothenbücher, J. Li, D. Sirkin, B. Mok, and W. Ju. Ghost driver: A field study investigating the interaction between pedestrians and driverless vehicles. In 25th IEEE International Symposium on Robot and Human Interactive Communication, RO-MAN 2016, pages 795-802, 2016.

24. L. Sorokin, N. Kauffmann, and R. Chadowitz. A change of perspective: Designing the automated vehicle as a new social actor in a public space. In CHI'19 Extended AbstractsCHI Conference on Human Factors in Computing Systems Extended Abstracts (CHI'19 Extended Abstracts), 2019.

25. F. Walker, D. Dey, M. Martens, B. Pfleging, B. Eggen, and J. Terken. Feeling-of-Safety Slider : A Platform for Measuring Pedestrian Comfort in Field Interactions with Vehicles. In Proceedings of the $2019 \mathrm{CHI}$ Conference on Human Factors in Computing Systems, pages 1-6, Glasgow, UK, May 2019.

\section{Bionotes}

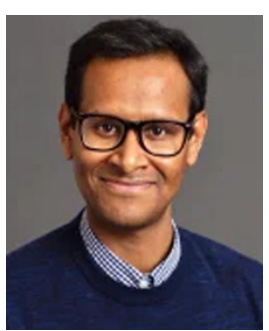

\section{Debargha Dey}

Eindhoven University of Technology, Groene Loper 3, 5612 AE Eindhoven, The Netherlands

d.dey@tue.nl

Debargha Dey is a doctoral candidate at Eindhoven University of Technology, Netherlands. He has a background in Human-Computer Interaction and his research interest lies in automotive human factors. His current doctoral work focuses on Human-Machine Interfaces for automated driving, and he is an active member of the $\mathrm{CHI}$ and AutomotiveUl communities.

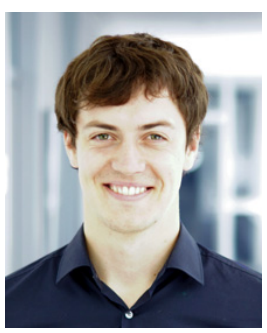

\section{Dr. Andrii Matviienko}

Technical University of Darmstadt, Hochschulstraße 10, 64289 Darmstadt, Germany matviienko@tk.tu-darmstadt.de

Andrii Matviienko is a postdoctoral researcher at Technical University of Darmstadt, Germany. His research focus lies on the assisting and educational technology for children and people with special needs. His past research focused on ambient light displays for car navigation and tangible awareness systems for working colleagues. 


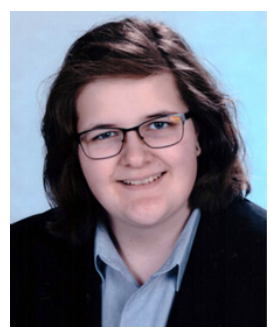

\section{Melanie Berger}

Eindhoven University of Technology, Groene Loper 3, 5612 AE Eindhoven, The Netherlands

m.berger@tue.nl

Melanie Berger is a doctoral candidate in the department of Industrial Design at Eindhoven University of Technology. She has a background in Human-Centered Computing and Informatics, and her current research interests lie in the domain of automotive human factors, particularly, novel in-car interaction concepts and the user experience of driving.

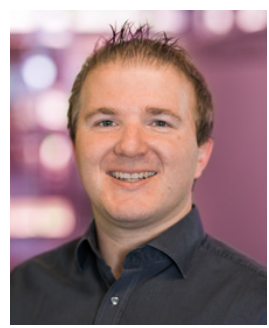

\section{Dr. Bastian Pfleging}

Eindhoven University of Technology, Groene Loper 3, 5612 AE Eindhoven, The Netherlands

b.pfleging@tue.nl

Bastian Pfleging is an Assistant Professor for Future Mobility at Eindhoven University of Technology, Netherlands. His research interests are user interfaces for future mobility, multimodal interaction, workload, and non-driving-related activities. He is actively involved in organizing conferences like CHI, AutomotiveUI, UIST, Mobile $\mathrm{HCl}$ and is steering committee member of AutomotiveUI.

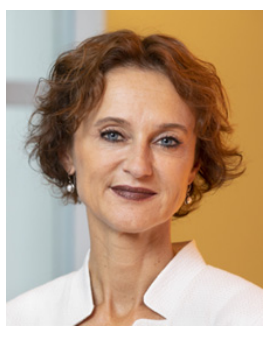

Prof. Dr. Marieke Martens

Eindhoven University of Technology, Groene Loper 3, 5612 AE Eindhoven, The Netherlands

m.h.martens@tue.nl

Marieke Martens is a Professor of Automated Driving and Human Interaction at the Eindhoven University of Technology and Science Director Traffic \& Transport at TNO, the Netherlands. She is an international expert in the areas of Human Factors, traffic safety, vehicle automation and behaviour, and has been working in this field, nationally and internationally for 24 years.

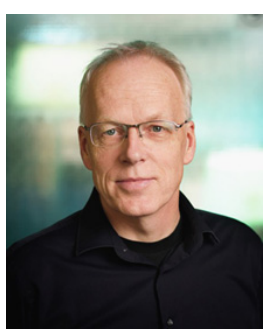

\section{Dr. Jacques Terken}

Eindhoven University of Technology, Groene Loper 3, 5612 AE Eindhoven, The Netherlands j.m.b.terken@tue.nl

Jacques Terken is an emeritus associate professor at Eindhoven University of Technology, the Netherlands. He has worked in the area of speech technology and human-technology interaction, and his current research focuses on automotive human factors. His research interests include the user experience for autonomous vehicles, and methods for evaluating design concepts in the early phases of the design process. He is the (co-)author of over 160 scientific papers covering topics such as the use of speech in the interface, usercentred design and evaluation, and automotive human factors. 